\title{
Management of systemic sclerosis-associated interstitial lung disease in the current era
}

Interstitial lung disease (ILD) is a group of diseases describing thickening of the interstitium surrounding pulmonary alveolar walls due to underlying inflammation. ILD is often associated with connective tissue diseases. Among connective tissue disease-related ILDs, $80 \%$ of systemic sclerosis (SSc) patients develop ILD ${ }^{1}$ with associated morbidity and mortality, ${ }^{2}$ although a large proportion may not develop clinically significant disease. Screening, early diagnosis and treatment of SSc-associated ILD (SSc-ILD) is important as up to $33 \%$ of SSc-related deaths are attributed to ILD ${ }^{3}$ and initiating treatment early in the course of the disease has been shown to slow disease progression. ${ }^{4,5}$ This article summarizes the risk factors for SSc-ILD and best practices for diagnosis and treatment of SSc-ILD.

\section{1 | RISK FACTORS AND SCREENING}

The goal of treatment in SSc-ILD is prevention of progression where progression is defined as new or worsening symptoms, increase in the extent of pulmonary fibrosis on high-resolution computed tomography (HRCT) and/or by a decline in pulmonary function tests (PFTs). ${ }^{6,7}$ This highlights the need for identifying patients at risk of disease progression before worsening occurs. The risk factors for progressive ILD are shown in Table 1. Although no absolute risk factors have been identified, these risk factors have been reported to be associated with progressive ILD in patients with SSc.

The risk of developing ILD is greatest early in the course of the disease and the most rapid decline in lung function (forced vital capacity [FVC]) occurs within the initial 3-5 years of disease onset. ${ }^{5}$ In addition, the extent of lung involvement on HRCT at baseline combined with reduced or declining FVC and diffusing capacity of the lung for carbon monoxide (DLCO) is predictive of mortality. ${ }^{6,8-10}$ Currently, the diagnosis of SSc-ILD is based on characteristic findings of increase reticulations with or without ground glass opacity on HRCT of the chest, irrespective of PFT results. Therefore, it is recommended that all patients with SSc receive HRCT at baseline. ${ }^{11,12}$ Additionally, all patients should be evaluated for evidence of cardiac involvement including assessment for pulmonary hypertension at the initial visit. ${ }^{13}$

Due to concern for radiation exposure with repeated HRCT, evaluation of lung function via spirometry and DLCO is recommended every 4-6 months in the first 3-5 years of the disease onset as this has been shown to provide valuable information about disease trajectory. ${ }^{6,14,15}$

\section{2 | TREATMENT}

The concepts for management of autoimmune ILD, including SSCILD, is shown in Figure 1. Not every patient with SSc-ILD requires treatment. Since early SSc-ILD is driven by immune activation and inflammation, the current approach involves the use of immunosuppressive therapy with the goal of initiation early in the disease to prevent advanced fibrotic disease. ${ }^{16}$ Our group has recently published single-center recommendations on the management of SSC-ILD. ${ }^{17}$ One strategy is to stratify patients by their lung disease severity (subclinical [defined as minimal ILD on HRCT, normal or near normal FVC and DLCO with no symptoms attributable to ILD] vs clinical ILD). For those with subclinical ILD, one may initiate therapy for ILD, especially with high-risk features or monitor closely for progressing symptoms, PFTs, and with repeat HRCT if necessary. ${ }^{17}$ Current practice involves treating patients on a case by case basis and only after careful review of risks vs benefits, as there is significant toxicity associated with some of the available medications.

The treatment for SSc-ILD has included the use of immunosuppressive therapies, in particular cyclophosphamide (CYC) and mycophenolate mofetil (MMF). MMF is largely used in North America and the UK for the management of SSc-ILD and it is generally given as $3000 \mathrm{mg} / \mathrm{d}$ in divided doses. This is based on the Scleroderma Lung Study II which showed that treatment with MMF offers comparable efficacy as oral CYC and has a better safety profile. ${ }^{18}$

Recent data from a Phase 3 trial showed that the treatment of early ILD (mean FVC\% of $82 \%$ with mild ILD on HRCT) in high-risk populations (early diffuse cutaneous SSc and elevated C-reactive protein [CRP] with $50 \%$ having positive anti-SCL-70 antibodies) with tocilizumab led to stabilization of FVC\% vs a decline of $6.5 \%$ in the placebo group at 48 weeks. ${ }^{19}$ Similarly, an open-label trial of patients with early diffuse cutaneous SSc and positive anti-SCL-70 found improvement in FVC\% at 24 weeks with the use of two courses of rituximab (1000 mg $\times 2$ doses) vs monthly pulse CYC. ${ }^{20}$ We offer treatment to patients with subclinical ILD and elevated CRP and/or positive anti-SCL-70.

Other therapy includes autologous hematopoietic stem cell transplantation (HSCT). Several trials have shown that HSCT following immunosuppressives has a beneficial effect in some patients with diffuse cutaneous SSc with multi-organ involvement. ${ }^{21}$ The appropriate patient has early disease with progressive ILD and is not responding to immunosuppressive therapy. The goal is to target aggressive and reversible disease. 
TABLE 1 Risk factors for progressive interstitial lung disease in systemic sclerosis ${ }^{\mathrm{a}}$

\begin{tabular}{l} 
Demographics \\
Male gender \\
African-American ethnicity \\
Disease type \\
Diffuse cutaneous SSc \\
Biomarkers \\
Anti-SCL-70/anti-topoisomerase Ab \\
Anti-nucleolar Ab (representing AntiTh/To, U3RNP) \\
C-reactive protein/interleukin-6 \\
Chemokine (C-C motif) ligand (CCL-18) \\
Krebs von den Lungen protein (KL6) ${ }^{\mathrm{b}}$ \\
Surfactant protein D (SP-D) \\
\hline
\end{tabular}

Abbreviations: Anti-SCL 70, anti-scleroderma-70 antibody; SSc, systemic sclerosis.

${ }^{a}$ Recently reviewed in Khanna et al. ${ }^{1}$

${ }^{\mathrm{b}} \mathrm{CCL}-18, \mathrm{KL} 6$ and SP-D are serum markers for endothelial injury.

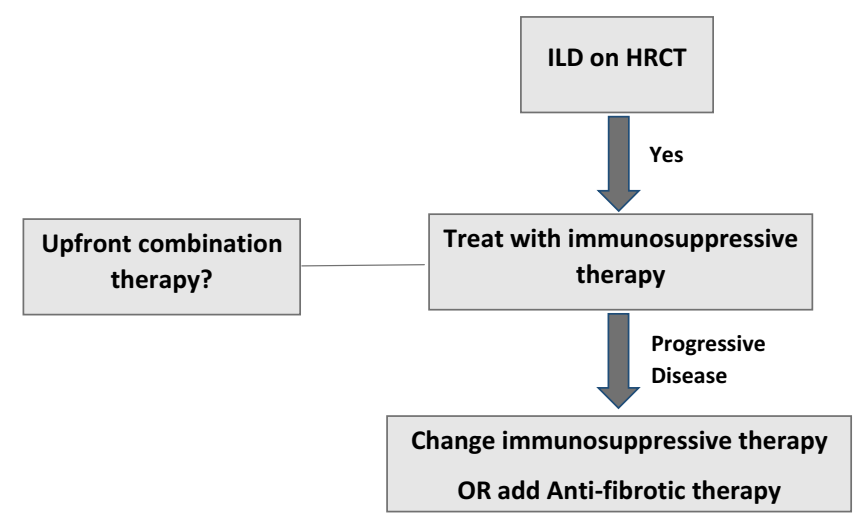

FIGURE 1 Overview of management of systemic sclerosisassociated interstitial lung disease. HRCT, high-resolution computed tomography; ILD, interstitial lung disease

As shown in Figure 1, a clinician may consider anti-fibrotic therapies in patients with progressive ILD or those intolerant of immuno-modulatory therapy (eg, due to recurrent infections). The SENSCIS trial, a large Phase 3 trial, demonstrated a smaller decline in FVC with the addition of nintedanib (an intracellular multiple tyrosine kinase inhibitor) to background MMF or no therapy vs placebo. ${ }^{22}$ Based on published data, sequential combination therapy could be considered for patients with high risk of progression (not addressed in the SENSCIS trail), where progression has occurred under monotherapy, or contraindication to the immunosuppressive therapy.

In addition to the above, comorbid conditions including infections and gastrointestinal disease/gastro-esophageal reflux disease (GERD) leading to chronic aspiration should be considered in patient with SSc-ILD. In addition to treatment of GERD, appropriate immunization, supplemental oxygen to keep saturations above $88 \%$, and education on tobacco cessation should be part of every patient's ongoing care. Lastly, pulmonary rehabilitation may be effective for some patients. ${ }^{23}$

\section{3 | CONCLUSION/SUMMARY}

Aside from autologous hematopoietic stem cell transplant, there is limited evidence to support a mortality benefit among SSc-ILD treatments and the benefits of all treatment strategies including combination therapies will need to be weighed against their side effects. Current management includes immunosuppressive therapy in those with subclinical ILD with high-risk features or those with clinical ILD. Anti-fibrotics should be considered in those with progressive ILD despite being on immunosuppressive therapy or if there is contraindication to immunosuppressive therapy. The ongoing trial Scleroderma Lung Study III is assessing if upfront combination of MMF and pirfenidone (anti-fibrotic) is more efficacious than MMF alone in early SSc-ILD (https://medicine.umich.edu/ sites/default/files/content/downloads/SclerodermaLungStudiesAugust2019-Final.pdf). Future research in SSc-ILD should aim at developing tools to identify patients at risk of progressive SSc-ILD and assess appropriate timing and treatment sequences in patients with SSc.

\section{FUNDING INFORMATION}

Dinesh Khanna, MD, MSc is supported by NIH/NIAMS K24 AR063120 and NIH/NIAMS R01 AR-07047.

Sogol Sara Amjadi ${ }^{1}$

David Roofeh ${ }^{2}$

Rajaie Namas ${ }^{3}$ iD

Dinesh Khanna²

${ }^{1}$ Dignity Health, Henderson, NV, USA

${ }^{2}$ Scleroderma Program, University of Michigan, Ann Arbor, MI,

${ }^{3}$ Division of Rheumatology, Cleveland Clinic, Ab Dhabi, United Arab Emirates

Correspondence

Dinesh Khanna, Department of Internal Medicine, Division of Rheumatology, 300 North Ingalls St., Suite 7C27, Ann

Arbor, MI 48109-5422, USA.

Email: khannad@med.umich.edu

\section{ORCID}

Rajaie Namas (iD https://orcid.org/0000-0003-0353-895X

Dinesh Khanna iD https://orcid.org/0000-0003-1412-4453

\section{REFERENCES}

1. Khanna D, Tashkin DP, Denton CP, Renzoni EA, Desai SR, Varga J. Aetiology, Risk Factors, and Biomarkers in Systemic Sclerosis with Interstitial Lung Disease. Am J Respir Crit Care Med. 2019. https://doi.org/10.1164/rccm.201903-0563Cl

2. Khanna D, Seibold JR, Wells A, et al. Systemic sclerosis-associated interstitial lung disease: lessons from clinical trials, outcome measures, and future study design. Curr Rheumatol Rev. 2010;6(2):138-144. 
3. Steen VD, Medsger TA. Changes in causes of death in systemic sclerosis, 1972-2002. Ann Rheum Dis. 2007;66(7):940-944.

4. Iudici M, Moroncini G, Cipriani P, Giacomelli R, Gabrielli A, Valentini G. Where are we going in the management of interstitial lung disease in patients with systemic sclerosis? Autoimmun Rev. 2015;14(7):575-578.

5. Steen VD, Medsger TA. Severe organ involvement in systemic sclerosis with diffuse scleroderma. Arthritis Rheum. 2000;43(11):2437-2444.

6. Goh NS, Hoyles RK, Denton CP, et al. Short-term pulmonary function trends are predictive of mortality in interstitial lung disease associated with systemic sclerosis. Arthritis Rheumatol Hoboken NJ. 2017;69(8):1670-1678.

7. Kim HJ, Tashkin DP, Gjertson DW, et al. Transitions to different patterns of interstitial lung disease in scleroderma with and without treatment. Ann Rheum Dis. 2016;75(7):1367-1371.

8. Moore OA, Goh N, Corte T, et al. Extent of disease on high-resolution computed tomography lung is a predictor of decline and mortality in systemic sclerosis-related interstitial lung disease. Rheumatol Oxf Engl. 2013;52(1):155-160.

9. Goh NSL, Desai SR, Veeraraghavan S, et al. Interstitial lung disease in systemic sclerosis: a simple staging system. Am J Respir Crit Care Med. 2008;177(11):1248-1254

10. Moore OA, Proudman SM, Goh N, et al. Quantifying change in pulmonary function as a prognostic marker in systemic sclerosis-related interstitial lung disease. Clin Exp Rheumatol. 2015;33(4 Suppl 91):S111-116.

11. Bernstein EJ, Khanna D, Lederer DJ. Screening high-resolution computed tomography of the chest to detect interstitial lung disease in systemic sclerosis: a global survey of rheumatologists. Arthritis Rheumatol Hoboken NJ. 2018;70(6):971-972.

12. Hoffmann-Vold A-M, Fretheim $\mathrm{H}$, Halse $\mathrm{A}-\mathrm{K}$, et al. tracking impact of interstitial lung disease in systemic sclerosis in a complete nationwide cohort. Am J Respir Crit Care Med. 2019;200(10):1258-1266.
13. Frost A, Badesch D, Gibbs JSR, et al. Diagnosis of pulmonary hypertension. Eur Respir J. 2019;53(1):1801904.

14. Schoenfeld SR, Castelino FV. Evaluation and management approaches for scleroderma lung disease. Ther Adv Respir Dis. 2017;11(8):327-340.

15. Volkmann ER, Tashkin DP, Sim M, et al. Short-term progression of interstitial lung disease in systemic sclerosis predicts long-term survival in two independent clinical trial cohorts. Ann Rheum Dis. 2019;78(1):122-130.

16. Wells AU, Margaritopoulos GA, Antoniou KM, Denton C. Interstitial lung disease in systemic sclerosis. Semin Respir Crit Care Med. 2014;35(2):213-221.

17. Roofeh D, Jaafar S, Vummidi D, Khanna D. Management of systemic sclerosis-associated interstitial lung disease. Curr Opin Rheumatol. 2019;31(3):241-249.

18. Tashkin DP, Roth MD, Clements PJ, et al. Mycophenolate mofetil versus oral cyclophosphamide in scleroderma-related interstitial lung disease (SLS II): a randomised controlled, double-blind, parallel group trial. Lancet Respir Med. 2016;4(9):708-719.

19. Khanna D, Lin CJF, Kuwana M, et al. Efficacy and safety of tocilizumab for the treatment of systemic sclerosis: results from a phase 3 randomized controlled trial. Arthritis Rheumatol. 70 (Suppl 10).

20. Sircar G, Goswami RP, Sircar D, Ghosh A, Ghosh P. Intravenous cyclophosphamide vs rituximab for the treatment of early diffuse scleroderma lung disease: open label, randomized, controlled trial. Rheumatol Oxf Engl. 2018;57(12):2106-2113.

21. Walker UA, Saketkoo LA, Distler O. Haematopoietic stem cell transplantation in systemic sclerosis. RMD Open. 2018;4(1):e000533.

22. Distler O, Highland KB, Gahlemann M, et al. Nintedanib for systemic sclerosis-associated interstitial lung disease. N Engl J Med. 2019;380(26):2518-2528.

23. Dowman LM, McDonald CF, Hill CJ, et al. The evidence of benefits of exercise training in interstitial lung disease: a randomised controlled trial. Thorax. 2017;72(7):610-619. 